\title{
Correlation between Surface Morphology and Corrosion Behaviour of Ni-P- Graphene and Ni-P-Carbon Nanotube Composite Coatings.
}

\author{
Atul Meshram ${ }^{1}$, M. K. Punith kumar ${ }^{2}$ and Chandan Srivastava ${ }^{3}$ \\ ${ }^{1}$ IISc, Karnataka, India, ${ }^{2}$ POST DOC, Karnataka, India, ${ }^{3}$ IISc Bangalore, Bengaluru, Karnataka, India
}

It has been shown recently that incorporation of graphene and carbon nanotubes (CNTs) into conventional metallic coatings leads to significant enhancement in the corrosion resistance behaviour of the coatings [1]. This enhancement is primarily due to the inert and impermeable nature of graphene and hydrophobicity of the carbon nanotubes. The optimum volume fraction of these carbonaceous additives (in the coating matrix) which can yield the lowest corrosion rate however need to be determined for every composite coating system as a lower additive amount can lead to inhomogeneous microstructure which is detrimental for corrosion resistance and a larger additive amount can lead to detrimental galvanic coupling where the carbonaceous additive (present as major phase) acts as cathode and the metallic matrix (present as minor phase) acts as anode. On more important aspect that is the effect of incorporation of carbonaceous additives on the morphological evolution and corrosion behaviour is illustrated here.

Amorphous Ni-P coatings exhibit properties such as excellent corrosion and wear resistance. In this work the effect of incorporation of graphene and CNT on the morphology and electrochemical behaviour of Ni-Pgraphene and Ni-P-CNT composite coating was investigated. Ni-P-graphene and Ni-P-CNT composite coatings were electrodeposited over mild steel substrate using the watts bath. Graphene synthesis was done by the electrochemical exfoliation of graphite [2] and CNTs were synthesised using the chemical vapour deposition technique [3]. Different amounts of the functionalized graphene and CNTs were dispersed in the aqueous electrolyte bath to produce Ni-P-graphene and Ni-P-CNT composite coatings with different volume fractions of the additives. The samples were subjected to electrochemical corrosion analysis in 3.5wt $\% \mathrm{NaCl}$ electroactive media. Corrosion properties of the coatings determined from the Tafel polarization and electrochemical impedance spectroscopy methods revealed that the incorporation of graphene and CNTs led to a continuous enhancement in the corrosion resistance of the composite coatings up to a certain optimum addition. Excess incorporation of the additives beyond the optimum led to increase in the corrosion rate to values beyond the one exhibited by the pristine coatings.

For Ni-P-Graphene composite coatings, pristine coatings and composite coatings produced using electrolyte bath with $100 \mathrm{mg} / \mathrm{l}, 150 \mathrm{mg} / \mathrm{l}$ and $200 \mathrm{mg} / \mathrm{l}$ of dispersed graphene exhibited the $\mathrm{i}_{\text {corr }}$ values of 3.93 $\mu \mathrm{A} / \mathrm{cm}^{2}, 0.304 \mu \mathrm{A} / \mathrm{cm}^{2}, 2.06 \mu \mathrm{A} / \mathrm{cm}^{2}$ and $2.20 \mu \mathrm{A} / \mathrm{cm}^{2}$ respectively. It is very apparent from the representative scanning electron microscopy (SEM) images of the Ni-P-graphene composite coatings provided in Fig. 1 that the initial incorporation of graphene (from pristine graphene to the Ni-P-graphene coating produced from $100 \mathrm{mg} / \mathrm{l} \mathrm{of}$ graphene) led to reduction in the morphological roughness which led to enhancement in the corrosion resistance behaviour of the coatings. A higher addition of graphene (coatings produced from 150 and $200 \mathrm{mg} / \mathrm{l}$ of graphene), on the other hand, increased the morphological roughness and led to the reduction in the corrosion resistance behaviour of the coatings. Similar results were obtained in case of the Ni-P-CNT composite coatings. For Ni-PCNT composite coatings, pristine coatings and coatings produced using $5 \mathrm{mg} / \mathrm{l}, 20 \mathrm{mg} / \mathrm{l}$ and $40 \mathrm{mg} / \mathrm{l}$ exhibited the $\mathrm{i}_{\text {corr }}$ values of $3.01 \mu \mathrm{A} / \mathrm{cm}^{2}, 0.69 \mu \mathrm{A} / \mathrm{cm}^{2}, 1.62 \mu \mathrm{A} / \mathrm{cm}^{2}$ and $2.17 \mu \mathrm{A} / \mathrm{cm}^{2}$. Again, it can be observed from the representative SEM morphological images of the Ni-P and Ni-P-CNT composite coatings that coating produced from electrolyte with $5 \mathrm{mg} / \mathrm{l}$ of CNT has the smoothest morphology which yielded the lowest corrosion rate. Whereas the coating produced from the electrolyte with $40 \mathrm{mg} / \mathrm{l}$ of CNT produced the most rough and defective morphology which led to the highest corrosion rate. Both the examples clearly illustrate that in addition to the intrinsic properties (such as impermeability of graphene and hydrophobicity of CNTs) of the additives their optimum addition which produces a smooth and defect free morphology is essential to obtain high corrosion resistance performance of the coatings. An excess incorporation of the additives can be detrimental with `regard to the corrosion resistance of the composite coatings. 


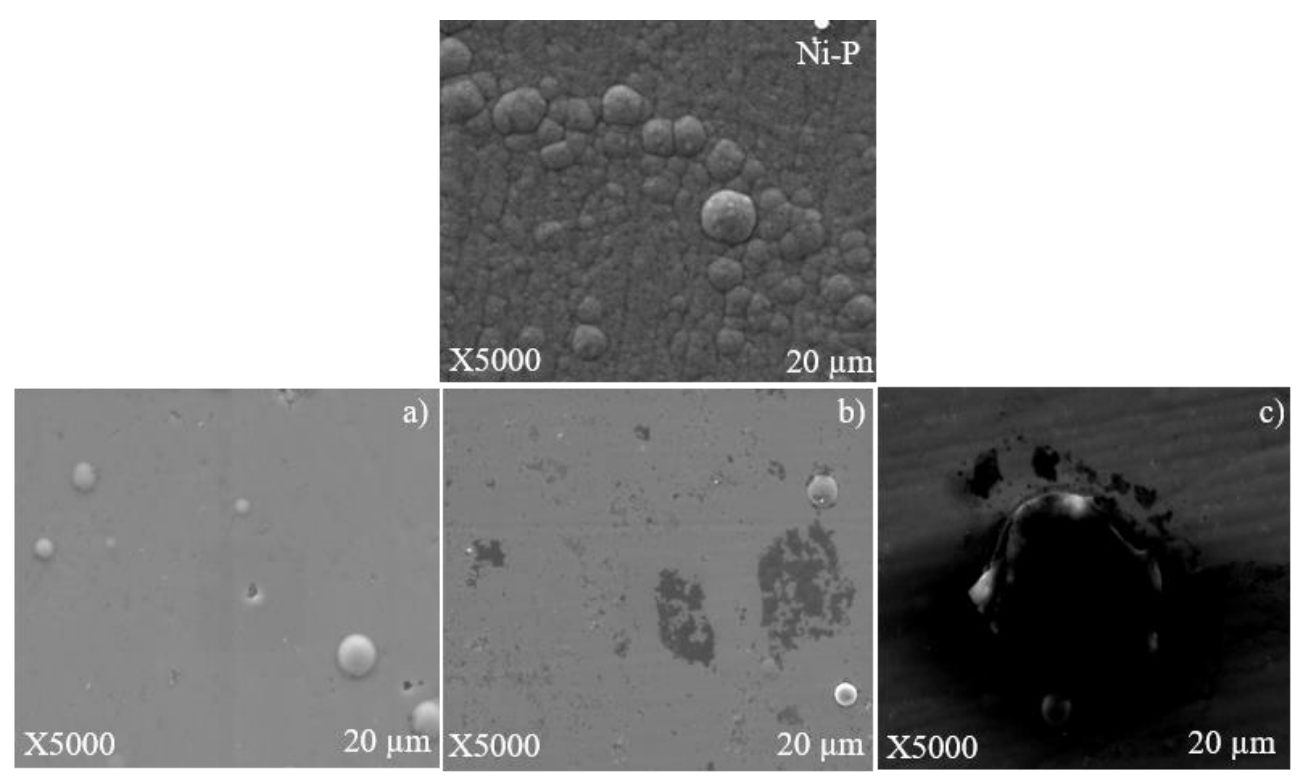

Figure 1. Figure. 1. High Mag SEM: Ni-P: Pristine Ni-P coatings; Ni-P-G composite coatings from bath composition a) 100mg/l graphene sample, b) 150mg/l graphene sample, c) 200mg/l graphene sample.
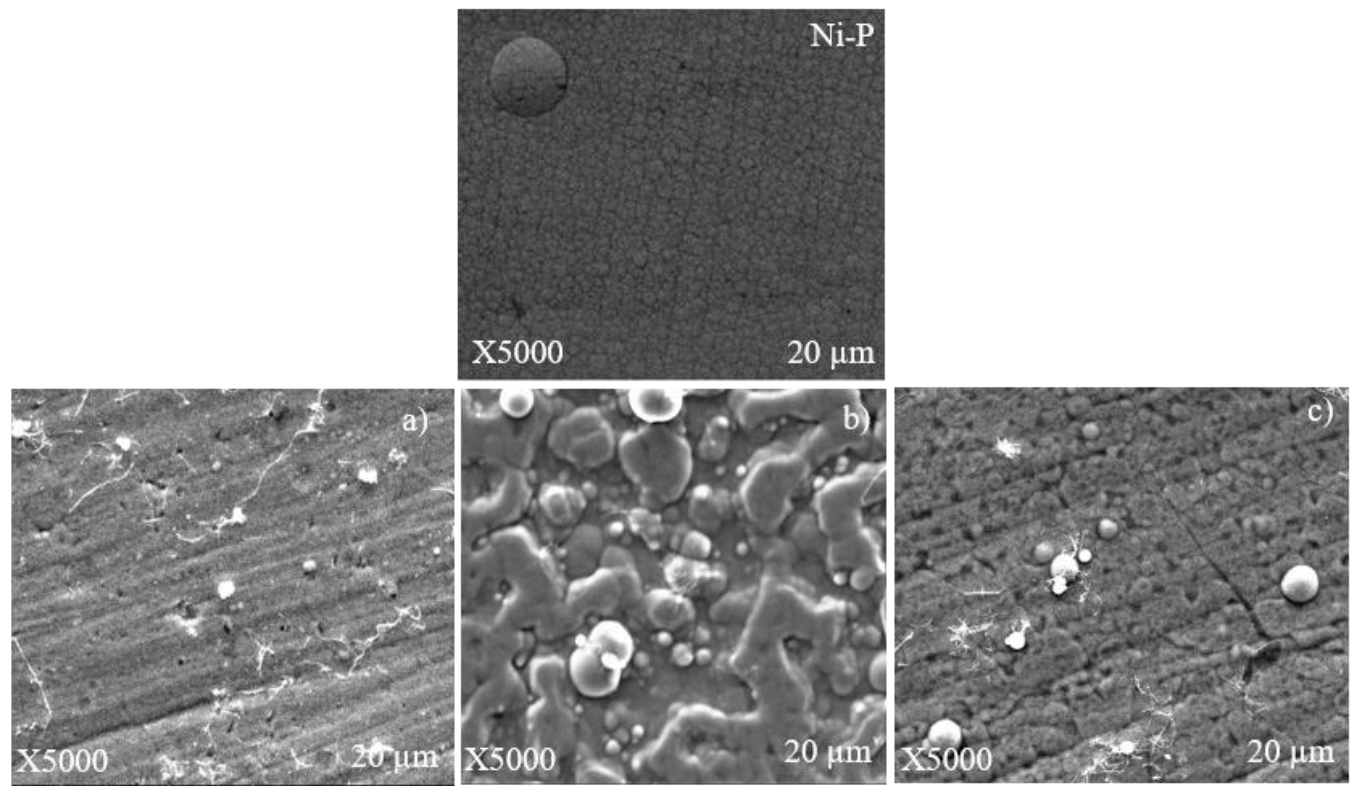

Figure 2. Figure. 2. High Mag SEM: Ni-P: Pristine Ni-P coatings; Ni-P-CNT composite coatings from bath composition a) 5mg/l CNT sample, b) 20mg/l CNT sample, c) 40mg/l CNT sample.

\section{References}

[1] M. K Punith Kumar, Rekha M. Y., Chandan Srivastava, "Electro galvanization Using New Generation Coatings with Carbonaceous Additives: Progress and Challenges" Corrosion Reviews.

[2] M. K. Punith Kumar, S. Shanthini, and Chandan Srivastava, "Electrochemical exfoliation of graphite for producing graphene using saccharin", RSC Adv., 2015, 5, 53865.

[3] Sweety Arora, Rekha M.Y, Abhay Gupta, and Chandan Srivastava, "High Corrosion Resistance offered by Multi-Walled Carbon Nanotubes Directly Grown Over Mild Steel Substrate” JOM 70(11), 2590(2018). 